\title{
A Single Disulfide Bond Disruption in the $\beta 3$ Integrin Subunit Promotes Thiol/Disulfide Exchange, a Molecular Dynamics Study
}

\author{
Lihie Levin ${ }^{1}$, Ehud Zelzion ${ }^{1,2 \infty}$, Esther Nachliel ${ }^{1}$, Menachem Gutman ${ }^{1}$, Yossi Tsfadia $^{1 *}$, Yulia Einav ${ }^{2 *}$ \\ 1 Biochemistry and Molecular Biology Department, George S. Wise Faculty of Life Sciences, Tel Aviv University, Tel Aviv, Israel, 2 Mathematical Biology Unit, Faculty of \\ Sciences, Holon Institute of Technology, Holon, Israel
}

\begin{abstract}
The integrins are a family of membrane receptors that attach a cell to its surrounding and play a crucial function in cell signaling. The combination of internal and external stimuli alters a folded non-active state of these proteins to an extended active configuration. The $\beta 3$ subunit of the platelet $\alpha$ llb $\beta 3$ integrin is made of well-structured domains rich in disulfide bonds. During the activation process some of the disulfides are re-shuffled by a mechanism requiring partial reduction of some of these bonds; any disruption in this mechanism can lead to inherent blood clotting diseases. In the present study we employed Molecular Dynamics simulations for tracing the sequence of structural fluctuations initiated by a single cysteine mutation in the $\beta 3$ subunit of the receptor. These simulations showed that in-silico protein mutants exhibit major conformational deformations leading to possible disulfide exchange reactions. We suggest that any mutation that prevents Cys560 from reacting with one of the Cys ${ }^{567}-C_{y} s^{581}$ bonded pair, thus disrupting its ability to participate in a disulfide exchange reaction, will damage the activation mechanism of the integrin. This suggestion is in full agreement with previously published experiments. Furthermore, we suggest that rearrangement of disulfide bonds could be a part of a natural cascade of thiol/disulfide exchange reactions in the $\alpha$ llb $\beta 3$ integrin, which are essential for the native activation process.
\end{abstract}

Citation: Levin L, Zelzion E, Nachliel E, Gutman M, Tsfadia Y, et al. (2013) A Single Disulfide Bond Disruption in the $\beta 3$ Integrin Subunit Promotes Thiol/Disulfide Exchange, a Molecular Dynamics Study. PLoS ONE 8(3): e59175. doi:10.1371/journal.pone.0059175

Editor: Junichi Sadoshima, University of Medicine and Dentistry of New Jersey, New Jersey Medical School, United States of America

Received December 18, 2012; Accepted February 12, 2013; Published March 18, 2013

Copyright: (c) 2013 Levin et al. This is an open-access article distributed under the terms of the Creative Commons Attribution License, which permits unrestricted use, distribution, and reproduction in any medium, provided the original author and source are credited.

Funding: Funding was provided by Tel-Aviv University special vice president support for Molecular Dynamics Research. The funders had no role in study design data collection and analysis, decision to publish, or preparation of the manuscript.

Competing Interests: The authors have declared that no competing interests exist.

*E-mail: YossiT@tauex.tau.ac.il (YT); yulia_e@hit.ac.il (YE)

a Current address: Department of Ecology, Evolution and Natural Resources, Rutgers University, New Brunswick, New Jersey, United States of America

\section{Introduction}

Integrins are a broad family of membrane-associated heterodimers that mediate any interaction between a cell and its surrounding tissues. The non-covalently associated trans-membrane $\alpha$ and $\beta$ integrin subunits contain both small intracellular and very large extracellular parts. The human platelet integrin $\alpha \mathrm{IIb} \beta 3$, also termed glycoprotein IIb/IIIa, is a typical representative of this wide family of cell adhesion receptors [1]. $\alpha \mathrm{IIb} \beta 3$ plays a crucial role in mediating platelet adhesion and aggregation by serving as a fibrinogen and a von Willebrand factor receptor. The binding of ligands to $\alpha \operatorname{IIb} \beta 3$ is strongly regulated; following activation by inside-out signals, the integrin goes through conformational changes resulting in ligand binding, clustering of the $\alpha \operatorname{IIb} \beta 3$ receptors, tyrosine phosphorylation and cytoskeleton rearrangements [2,3]. The precise mechanism of $\alpha \operatorname{IIb} \beta 3$ activation is still unknown, but the crystallographic data suggest equilibrium between a bent and an extended conformation, which is able to bind a ligand. Several intermediate affinity states have also been suggested [4-7], and an alternative model involving release of a "deadbolt" created by an interface of a two distant domains has also been proposed [8-10].
Both $\alpha \mathrm{IIb}$ and $\beta 3$ subunits of the protein contain many conserved cysteine residues, which form disulfide bonds. The extracellular $\beta 3$ subunit contains 56 highly conserved cysteines located in the four integrin-epidermal growth factor-like (I-EGF) repeats and a $\beta$-tail domain, situated in the membrane-proximal, "lower leg" region of the protein. Several studies have shown that the integrin activation process depends on the formation of free thiols with a subsequent disulfide exchange reaction [11-14]. Indeed, addition of dithiothreitol, a reducing agent, leads to global conformational changes and the opening of ligand binding site [15]. Integrin $\alpha \mathrm{IIb} \beta 3$ has also been shown to exhibit endogenous thiol isomerase activity [16]. Moreover, the active state of the integrin contains more free thiols than the resting conformation [17]. Therefore, the cysteine-rich region of $\beta 3$, which is located between the cytoplasmic domains and the ligand-binding headpiece, is purported to be the fulcrum of rearrangement events which are associated with the activation [18]. In accordance with this assumption, there are several natural mutations in cysteine residues that lead to the inherited bleeding disorder Glanzmann Thrombasthenia (GT), displaying a defective platelet aggregation. The phenotypes of the various mutations associated with this disorder range from the total absence of $\alpha \operatorname{IIb} \beta 3$ on the platelet membrane (due to abnormal conformation of $\beta 3$ ) to a constitu- 
tively active complex permanently locked in a high-affinity state [19-22]. Interestingly, some cysteine mutations in the same $\beta 3$ subunit affect the conformation and function of the $\alpha \operatorname{IIb} \beta 3$ and the closely related $\alpha_{v} \beta 3$ complexes differently [23]. How the disulfide isomerization and the free thiols integrate into the integrin activation models is still unresolved and the specific redox sites in the $\beta 3$ integrin subunit have not yet been described.

Each of the four I-EGF domains located in the "lower leg" of the $\beta 3$ subunit contain eight cysteines which, according to crystal structures, form four disulfide bonds in an order of: $1-5,2-4,3-6$ and 7-8 pattern (except for I-EGF1 that lacks the $2-4$ bond) $[24,25]$. The $1-5$ bond is unique for the EGF element of the integrins, whereas the $2-4,3-6$ and $7-8$ bonds are conserved in other EGF domains $[18,26]$. It should be noted that, according to a trypsin digestion assay, the pattern of pairing was slightly different, e.g. for the I-EGF4 domain, the 1-2 and 3-5 pairing were proposed [27]. Thus, it might be possible that under in vivo conditions the disulfide pattern is flexible and varies according to the physiologic state. Considering that for all published crystal structures of the complete ectodomain $[6,24,25,28]$ the integrins are proposed to be in their non-active state, it is possible that the active form of the receptor may contain free thiols and that disulfide rearrangement is an integral process of coupling the cytoplasmic tails with the large extracellular headpiece during integrin activation.

In this study we sought to investigate the outcome of disulfide bond opening by in-silico mutation of one of its cysteine residues using Molecular Dynamics (MD) simulations. The Cys ${ }^{560}-\mathrm{Cys}^{583}$ bond is the integrin-specific 1-5 pair located in the I-EGF4 domain $[18,26]$. The natural occurring mutation C560R was identified as the cause for the GT bleeding disorder and is coupled with a constitutively active state of the $\alpha \mathrm{IIb} \beta 3$ integrin. On the other hand, the artificial mutation, in which the other cysteine of the pair (Fig. 1) is replaced by serine (C583S), displays an almost normal state of activation [22-24]. Another important pair is the $\mathrm{Cys}^{567}-\mathrm{Cys}^{581}$ bond (2-4 pair), in which replacement of either the Cys567 or the Cys581 residues blocks the activation of the mutated protein [29]. In the present study we shall address the possibility that opening of such a disulfide bond may initiate structural rearrangements leading to the approach of a free thiol to an existing disulfide with subsequent disulfide isomerization. The question associated with such a reaction is whether the sequence of thiol/disulfide exchange events will be random or, the internal structure of the protein will impose a predetermined sequence of structural rearrangements, leading to regiospecificity.

The MD simulation method has been used successfully in several investigations of the $\alpha \operatorname{IIb} \beta 3$ structure [30-34]. However, these studies focused mainly on the headpiece region and the ligand-binding site. In the present study we investigate a single disulfide bond disruption in the "lower leg" region of the $\beta 3$ subunit. We show structural consequence of bond opening and demonstrate that in some cases it enables a thiol/disulfide exchange reaction of the free thiol with another cysteine pair. An artificial exchange reaction, followed by additional MD simulations, reveals proteins that strongly prefer defined conformational states. To our best knowledge, this is the first time that specific thiol/disulfide exchange events in the $\alpha \operatorname{IIb} \beta 3$ integrin have been projected by computational technology.

\section{Materials and Methods}

\section{Molecular Dynamics Simulations}

The atomic coordinates of the I-EFG1, I-EFG2, I-EFG3, IEFG4 and $\beta$-tail fragment ( $\beta 3$ subunit residues: 436-690) of integrin $\alpha \mathrm{IIb} \beta 3$ were obtained from the Protein Data Bank (PDB ID: 3FCS) [25]. Completion of the six missing residues (477-482) in the 3FCS protein structure and implementation of the point mutations (C583S, C560R and C581S) were performed using the Swiss-PDB Viewer program [35].

Table 1 presents a list of the simulations discussed in our study. The wild type 1 (WT-1), C583S and C560R MD simulations were performed using GROMACS 3.3.3 suite of software [36], whereas the disulfide exchange MD simulations $\left(\mathrm{Cys}^{560}-\mathrm{Cys}{ }^{567}\right.$, $\mathrm{Cys}^{560}$ $\mathrm{Cys}^{581}$ and $\mathrm{Cys}^{567}{ }^{5} \mathrm{Cys}^{586}$, WT-2 and the C581S) were performed using GROMACS 4.07 [37]. All of the simulations were carried out using the GROMACS 53a6 force field [38].

The proteins were immersed in triclinic boxes filled with SPG [39] water molecules that extended to at least $12 \AA$ between the molecule and the edge of the box. $\mathrm{Na}^{+}$and $\mathrm{Cl}^{-}$ions were added to neutralize the system up to a physiological salt concentration of $100 \mathrm{mM}$. The whole system - protein, water and ions - was energy-minimized using the steepest descent algorithm with a force tolerance of $1000 \mathrm{~kJ} \mathrm{~mol}^{-1} \mathrm{~nm}^{-2}$. A $40 \mathrm{ps}$ simulation with position restraints was performed at $300 \mathrm{~K}$ to "soak" the water molecules into the protein. The system was simulated further for a 1 ns unrestrained equilibration simulation. Starting with the final structure of the preliminary simulation, the MD simulation was set up. Considering that our simulations were limited to a section of the protein, position restraint was applied on the edges of the protein in order to mimic the natural constraint on these atoms in the intact protein. Varying force intensities were applied between simulations, trying to reach maximal flexibility without losing the basic structure of the protein. The initial force tested in our study was $1000 \mathrm{~kJ}$ $\mathrm{mol}^{-1} \mathrm{~nm}^{-2}$ applied on all heavy atoms of the first residue of the $\mathrm{N}$ terminus and on all heavy atoms of the last two residues of the protein on the $\mathrm{C}$ terminus. Under this force the protein was too rigid, thus in the succeeding simulations the force was applied only on the first heavy atom of I-EGF1 and the last heavy atom of the $\beta$-tail domain. Finally, to attain maximal conformational changes we reduced the force applied on the atoms to $100 \mathrm{~kJ} \mathrm{~mol}^{-1} \mathrm{~nm}^{-2}$; this ensured maximal flexibility while the protein maintained its structure. Detailed information can be found in the supplementary data (Table S1).

The MD simulations were run under NPT (constant Number of particles, Pressure, and Temperature) conditions using Berendsen's coupling algorithm for keeping the temperature and pressure constant $\left(\mathrm{P}=1\right.$ bar; $\left.\tau_{\mathrm{p}}=0.5 \mathrm{ps} ; \mathrm{T}=300 \mathrm{~K} ; \tau_{\mathrm{R}}=0.1 \mathrm{ps}\right) \quad[40]$. During the runs, the LINCS (Linear Constraint Solver) algorithm [41] was used to constrain the lengths of all bonds; the water molecules were restrained using the SETTLE algorithm. A $12 \AA$ cutoff was used for the Van der Waals interactions. The longrange electrostatic interactions were treated by the Particle Mesh Ewald method [42]. The coordinates were saved every 1 or 2 ps. The MD simulation time-step was 2 fs.

\section{Trajectory Analysis}

Trajectories obtained from various simulations were analyzed using flexible tools provided by GROMACS. Visual Molecular Dynamics (VMD) was used for the visual analysis of the trajectories [43].

Cluster analysis was performed for the trajectories of the simulations by the command g_cluster of the GROMACS 4.07 package. Cluster analysis was applied using the Gromos algorithm with an RMSD (Root Mean Square Deviation) cut-off value of $0.4 \mathrm{~nm}$ [44]. 


\section{I-EGF3}

\section{I-EGF4}

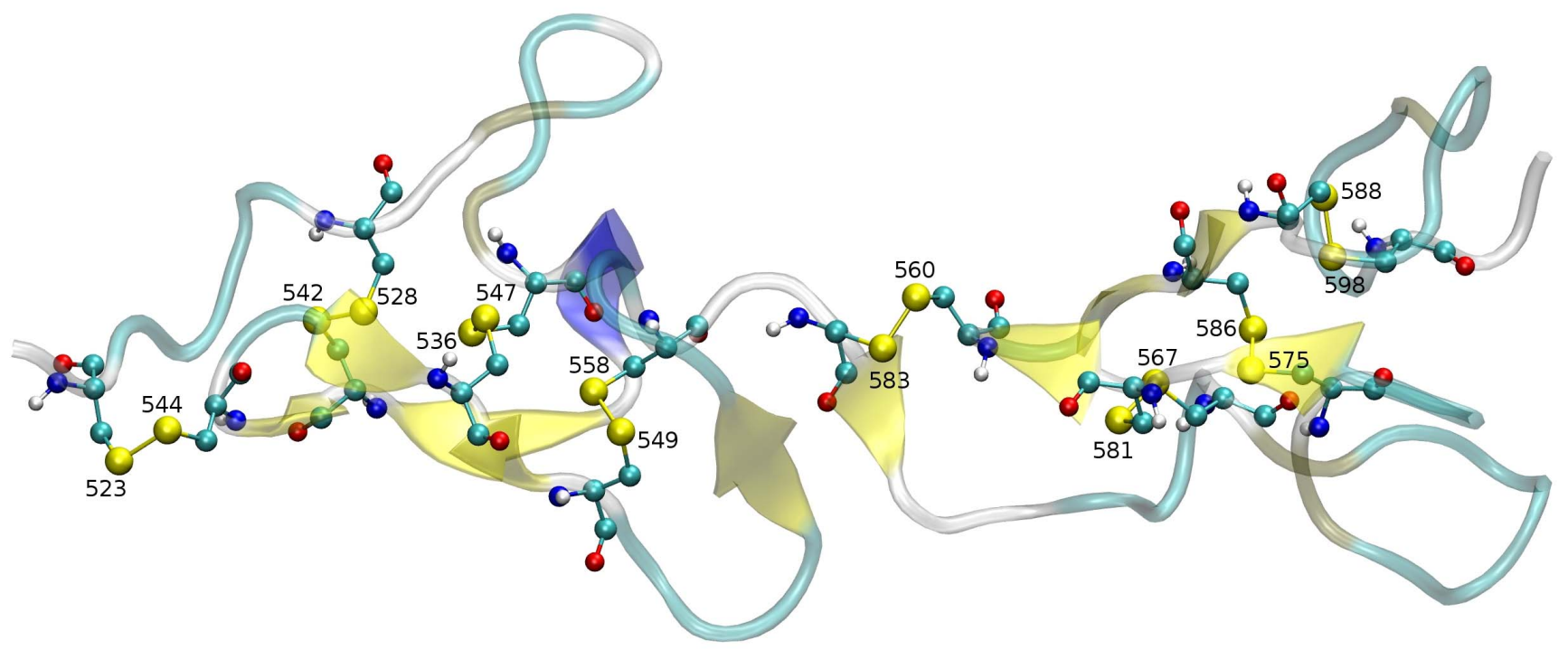

Figure 1. Disulfide network in I-EGF3 and I-EGF4. The figure is taken from the crystal structure, PDB code: 3FCS (27). Domains I-EGF3 and I-EGF4 are in cartoon representation; Cysteine residues are shown in balls-and-sticks and numbered as in the mature protein.

doi:10.1371/journal.pone.0059175.g001

\section{Disulfide Exchange}

Last frame of C583S simulation was saved and the free sulfur of the Cys560 was connected either to Cys581 or Cys567 and this structure used as an initial coordinate for two other $30 \mathrm{~ns}$ simulations, named $\mathrm{Cys}^{560}-\mathrm{Cys}^{581}$ and $\mathrm{Cys}^{560}-\mathrm{Cys}^{567}$, respectively (Table 1). For the Cys ${ }^{567}-\mathrm{Cys}^{581}$ exchange simulation the last frame of C581S run was saved and the free sulfur of the Cys567 was connected to the Cys586 and simulated for extra $40 \mathrm{~ns}$ (Table 1, Gys ${ }^{567}-\mathrm{Cys}^{586}$ ).

\section{Disulfide Pairing in the 3FCS Crystal}

WT disulfide pairing in the I-EGF4 domain of the integrin $\alpha \mathrm{IIb} \beta 3$ as resolved in the 3FCS crystal [25] (Cys ${ }^{560}-\mathrm{Cys}^{583}$, $\mathrm{Cys}^{567}-\mathrm{Cys}^{581}, \mathrm{Cys}^{575}-\mathrm{Cys}^{586}, \mathrm{Cys}^{588}-\mathrm{Cys}{ }^{598}$ ) is detailed in Figure 1.

\section{Results}

The Structural Fluctuation of the Simulated Proteins

The WT protein and the in-silico created C583S mutant of the $\beta 3$ subunit were subjected to MD simulations for 35 ns. Panels $A$ and $B$ of Figure 2 show superposition of the starting conformation of the I-EGF1 to $\beta$-tail fragment with the most popular conformations of the WT (Fig. 2A) and of C583S (Fig. 2B) accepted along the MD runs. The superposition images of the individual domains are represented in the upper panel of Figure 2. Inspection of the alignment of the starting structure with the simulated WT reveals that the lower part of the $\beta 3$ subunit maintains a stable structure along the simulation time, both for the individual domains and the entire structure alignments (Fig. 2A and upper panels). This indicates that a simulation of a protein fragment, without introducing any changes to its primary native structure, keeps its conformation at around the starting point. For the C583S mutant, the superposition of the entire structure

Table 1. List of simulations of integrin $\alpha$ llb $\beta 3$, discussed in the present study.

\begin{tabular}{|c|c|c|c|c|}
\hline & Simulation & Free Thiol & Total Simulation time (ns) & Simulation Type \\
\hline 1 & WT & None & $70^{\mathrm{a}}$ & Wild type protein \\
\hline 2 & C560R & Cys583-SH & 35 & In silico mutation \\
\hline 3 & C583S & Cys560-SH & $341^{\mathrm{a}}$ & In silico mutation \\
\hline 4 & $\mathrm{Cys}^{560}-\mathrm{Cys}^{567}$ & Cys581-SH & 35 & $\begin{array}{l}\text { In silico thiol/disulfide exchange } \\
\text { product of C583S mutation }\end{array}$ \\
\hline 5 & $\mathrm{Cys}^{560}-\mathrm{Cys}^{581}$ & Cys567-SH & 35 & $\begin{array}{l}\text { In silico thiol/disulfide exchange } \\
\text { product of } \mathrm{C} 583 \mathrm{~S} \text { mutation }\end{array}$ \\
\hline 6 & C581S & Cys567-SH & $90^{\mathrm{a}}$ & In silico mutation \\
\hline 7 & $\mathrm{Cys}^{567}-\mathrm{Cys}^{586}$ & Cys575-SH & 40 & $\begin{array}{l}\text { In silico thiol/disulfide exchange } \\
\text { product of } \mathrm{C} 581 \mathrm{~S} \text { mutation }\end{array}$ \\
\hline
\end{tabular}

a2 simulations of WT protein, 7 of C583S (with different restrain conditions; for more details see Table S1) and 3 of C581S were conducted.

doi:10.1371/journal.pone.0059175.t001 


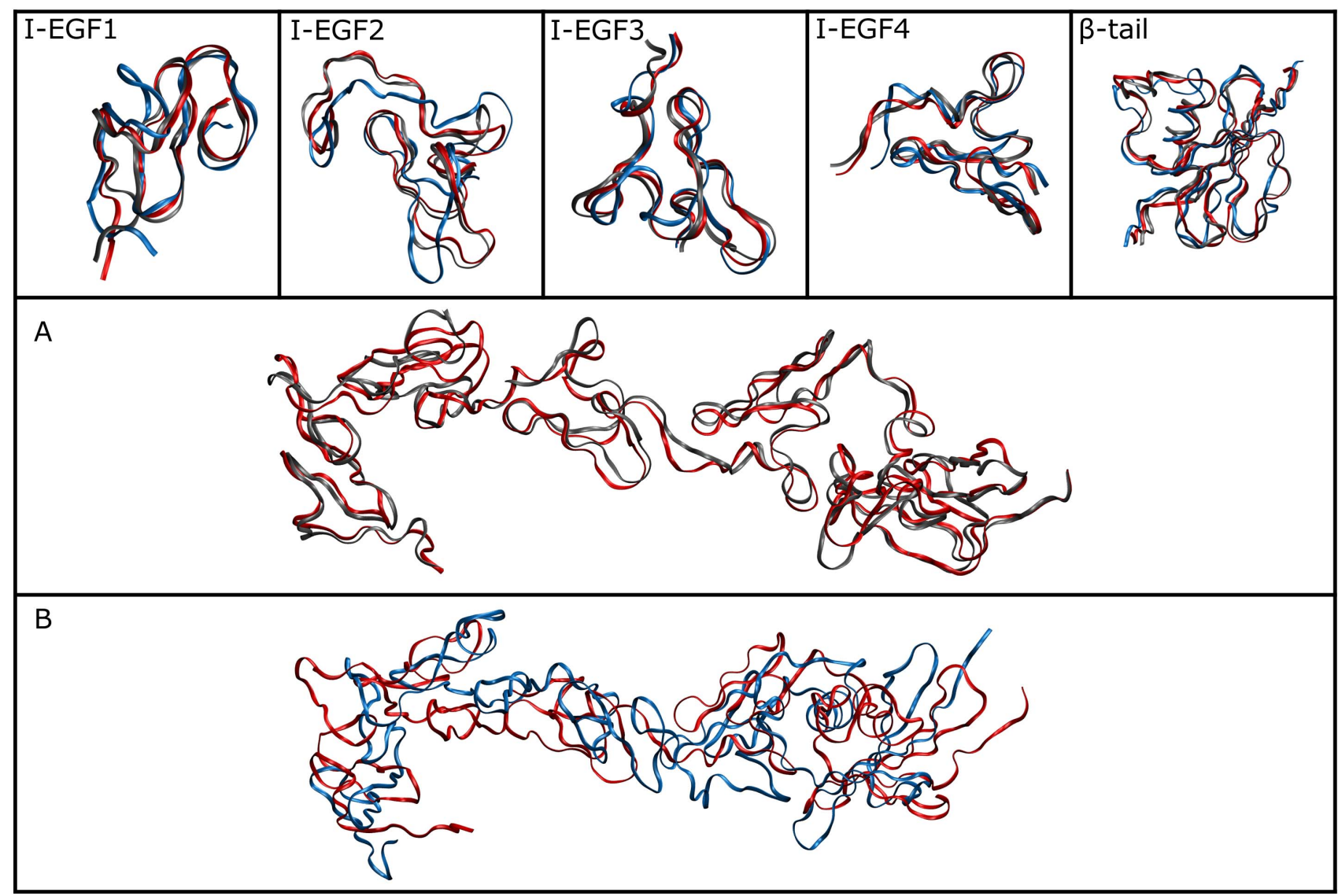

Figure 2. Superposition of the initial and the simulated structures. A - Superposition of the initial structure (red) vs. the most favorable conformation of a simulated WT protein (gray). B - Superposition of the initial structure (red) vs. the most favorable conformation of the simulated C583S mutant (blue). The upper panels introduce the differences in each domain (I-EGF1 to $\beta$-tail as indicated); the lower panels show the entire penta-domain structure.

doi:10.1371/journal.pone.0059175.g002

(Fig. 2B) shows that its global shape was grossly affected by the single mutation. However, no significant alterations in the rigid domain conformations were observed (Fig. 2 upper panels). Thus, the differences between the various structures sampled during the simulation are due to inter-domain movements.

In the C560R mutation, a phenotype characterized by over activation, a significant movement between the domains can be seen when aligning the entire structure. Yet, in contrast with the trajectory of C583S, in the case of C560R there is also a slight motion within the domains (Fig. S1).

The RMSD values for WT, C583S and also for C560R simulations are shown in Figure S2 in the Supporting Material.

The Distances between Free Thiols and Disulfide Bonds

The $\mathrm{Cys}^{560} \mathrm{Cyys}^{583}$ bond is positioned near the junction between IEGF3 and I-EGF4. Thus, two potential disulfide exchange events are optional; the first is with the Cys ${ }^{549}-\mathrm{Cys}^{558}$ pair located in I-EGF3 and the second is with the $\mathrm{Cys}^{567}-\mathrm{Cys}^{581}$ pair located in I-EGF4. To check the possibility of disulfide isomerization in cysteine mutants, we monitored the distance between the free cysteine (Cys583-SH or Cys560-SH) and the closest disulfide bonds during the simulation (Table 1, simulations 2 and 3).

Seven independent simulations of the "in-silico" C583S mutated protein, (Table S1, total $341 \mathrm{~ns}$, 188,000 frames) were analyzed and the number of frames in which the free thiol of Cys560 was within a certain distance range from the nearest disulfide bond was calculated (Fig. 3). In $18.2 \%$ of the total simulation frames, the free thiol of Cys560 was in very close proximity $(<0.4 \mathrm{~nm})$ to the Cys ${ }^{567}$ - Cys ${ }^{581}$ bond and in an additional $26.3 \%$ of the frames, the distance was within a range of $0.4-0.6 \mathrm{~nm}$. Overall, in $44.5 \%$ of total simulation times the distance between the free thiol and the disulfide bond was shorter than $0.6 \mathrm{~nm}$. Under physiologic conditions, once the sulfhydryl comes within such proximity to a disulfide bond, an exchange event is possible, with subsequent appearance of a new stable structure.

Figure 4 depicts the variation over time of the distance between the free thiol of Cys560 and the nearby disulfide pair, in a typical C583S simulation (Table S1, number 7). The results revealed a distinct preference for the $\mathrm{Cys}^{567}-\mathrm{Cys}^{581}$ pair (Fig. 4, left panel). At the beginning of the simulation, the free thiol of Cys560 was $\sim 10 \AA$ from this pair. Yet, within $10 \mathrm{~ns}$, the distance was drastically shortened, and after some fluctuations it finally stabilized at a distance range of $3.5-5.2 \AA$, until the end of the simulation time.

The naturally occurring C560R mutation is known to constitutively activate integrin. The simulation of this mutant revealed that the free thiol of Cys583 did not approach any of the nearest disulfide bonds and maintained an average distance of $8.5 \AA$ from the $\mathrm{Cys}^{567}-\mathrm{Cys}^{581}$ bond during the entire simulation time (Fig. 4, right panel). Apparently the asymmetric abolishment of a disulfide bond can lead to far-reaching consequences at the physiologic level. 


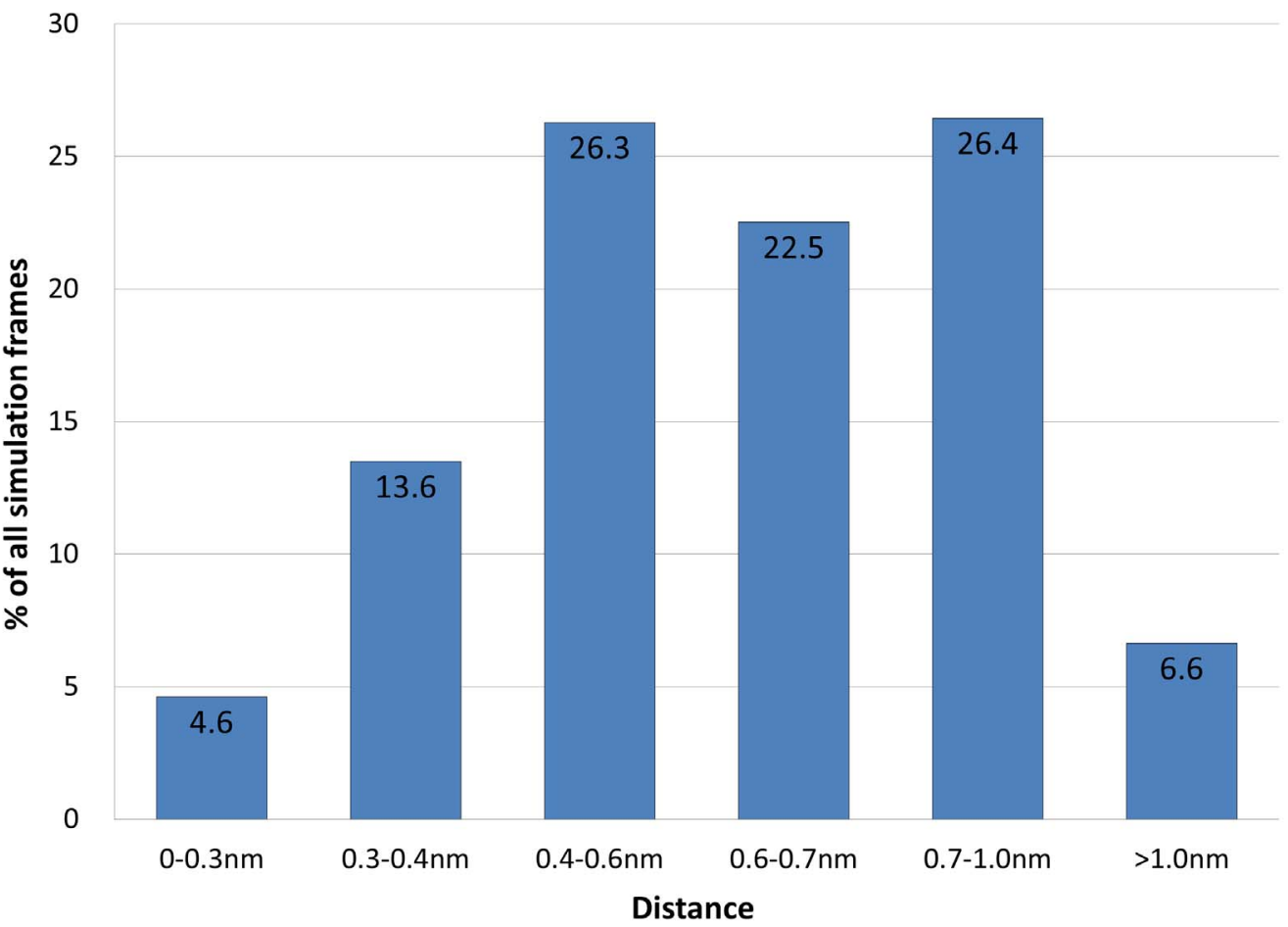

Figure 3. Distance range between a free thiol of Cys560 and a Cys567-Cys581 bond. The minimum distance between Cys560 free thiol and Cys567-Cys581 pair was calculated in all the seven C583S simulations. The number of frames in which the distance lies in a certain range was computed over a total of $\sim 188,000$ simulation frames and is presented inside the columns.

doi:10.1371/journal.pone.0059175.g003

It should be emphasized that in both trajectories (C560R and C583S) the free sulfhydryl groups made no attempt to make contact with any of the disulfide pair of I-EGF3 (see Figure S3). Thus, we can suggest that these mutations do not lead to a merging between the I-EGF3 and I-EGF4 domains of the $\beta 3$ subunit.

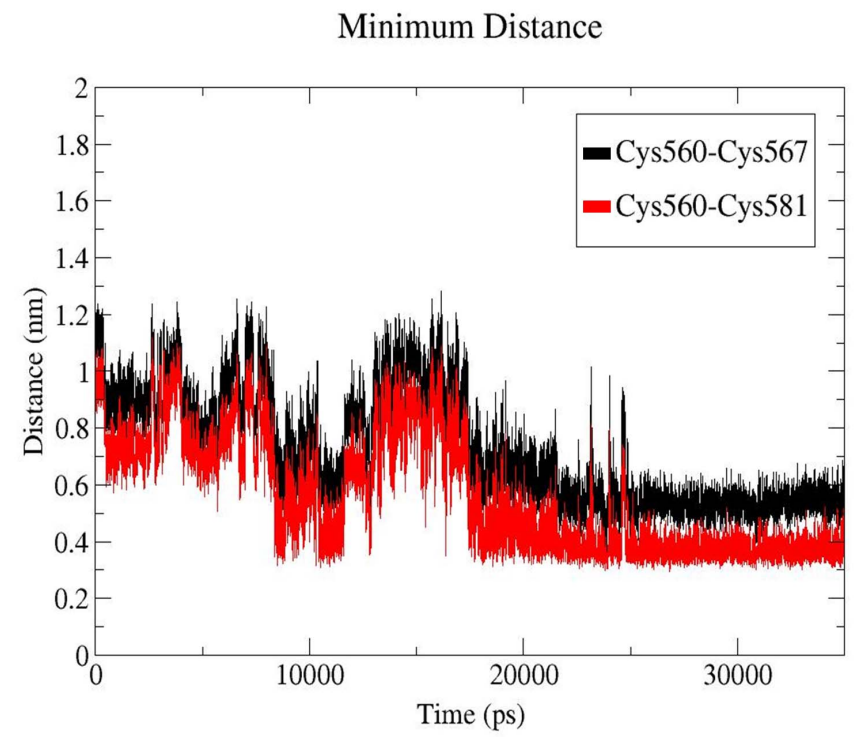

The Rearrangement of the Disulfide Network

In order to reveal the consequences of disulfide exchange events, the two feasible in-silico exchange reactions were generated, and simulated for $35 \mathrm{~ns}$. The free thiol of Cys560 was connected to either Cys567 or to Cys581, making new Cys ${ }^{560}-\mathrm{Cys}^{567}$ or $\mathrm{Cys}^{560}{ }^{5 y s}{ }^{581}$ disulfide bonds for the two consequent MD

Figure 4. Distance between disulfide pairs as a function of simulation time. Distance between sulfur atoms of the free thiol Cys560 in the representative C583S simulation and of the $\mathrm{Cys}^{567}-\mathrm{Cys}^{581}$ bond (left). Distance between sulfur atoms of the free thiol Cys583 in the C560R simulation and of the $\mathrm{Cys}^{567}-\mathrm{Cys}^{581}$ bond (right).

doi:10.1371/journal.pone.0059175.g004 
simulations (simulations 4 and 5, Table 1). Generally, the outcome of the simulations is not monolithic and the quantitative evaluation of the various structures is needed. To characterize the many conformational states sampled by the simulations of the WT, C583S and the two exchange products $\left(\mathrm{Cys}^{560}-\mathrm{Cys}^{567}\right.$ and $\mathrm{Cys}^{560}-\mathrm{Cys}^{581}$ ), cluster analysis was performed (Fig. 5).

The cluster analysis was carried out with a cut-off value of $0.4 \mathrm{~nm}$, a value that generated more than 10 clusters per simulation, each containing a sufficient number of configurations to attain statistical significance. The main panels in Figure 5 depict the sequence of the structural fluctuations that the protein undergoes, classifying the momentary structure according to the cluster type. Thus, in the WT trajectory the initial state corresponded with cluster \#3, with a brief sampling of the configurations corresponding with clusters \#10 and \#6. After $\sim 6 \mathrm{~ns}$, cluster \#2 became more prevalent and at $\sim 18 \mathrm{~ns}$ cluster \# 1 became the most popular. However, during the whole length of the trajectory the structure gained conformations compatible with the less popular clusters, as demonstrated by the sporadic association with clusters having a lower probability. The overall distribution between the clusters is given by the histogram inserted into the frame. Apparently, the WT protein has 4 major structures that consist of $93 \%$ of all configurations, where the difference in free energy between them (calculated as $\Delta \mathrm{G}=-\mathrm{k}_{\mathrm{B}} T \times \ln \frac{\text { cluster }_{i}}{\text { cluster }_{i}}$ ) is well below $\mathrm{k}_{\mathrm{B}} T$. Based on this value, we can assume that all structures are in equilibrium with rapid transitions between them.

The simulation of the C583S mutant reveals slightly higher fluctuations between clusters, as the two largest clusters are almost equal in size and the fraction of the 4 major clusters decreases from $93 \%$, as in the WT, to 75\% in C583S (Fig. 5, upper panels). Once the Cys560 is attached to either Cys567 or the Cys581, the protein gains relative stability (with respect to C583S where Cys560 has free thiol) and the largest cluster in both mutants is populated for $65 \%$ and $57 \%$ of the trajectories, respectively (Fig. 5, lower panels). These results clearly indicate that the disulfide exchange of the Cys560 free thiol with the Cys ${ }^{567}-\mathrm{Cys}^{581}$ bond stabilizes the
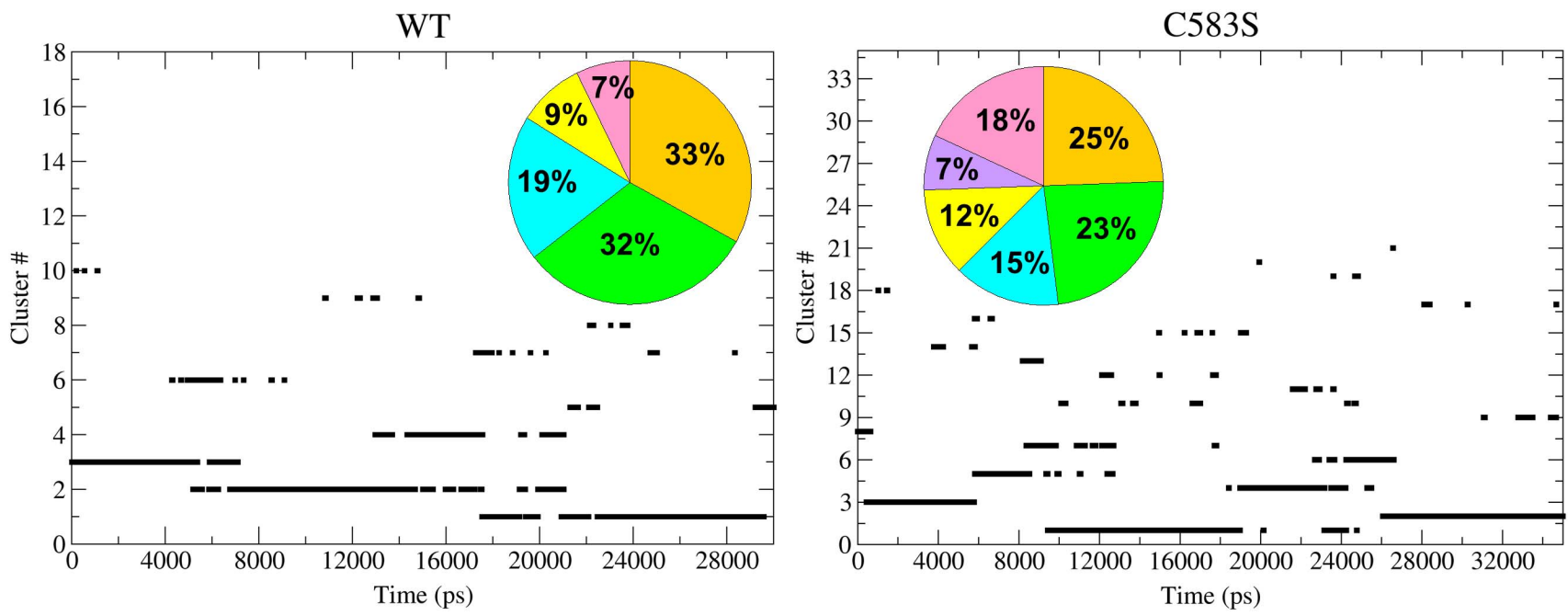

Exchange 560-567

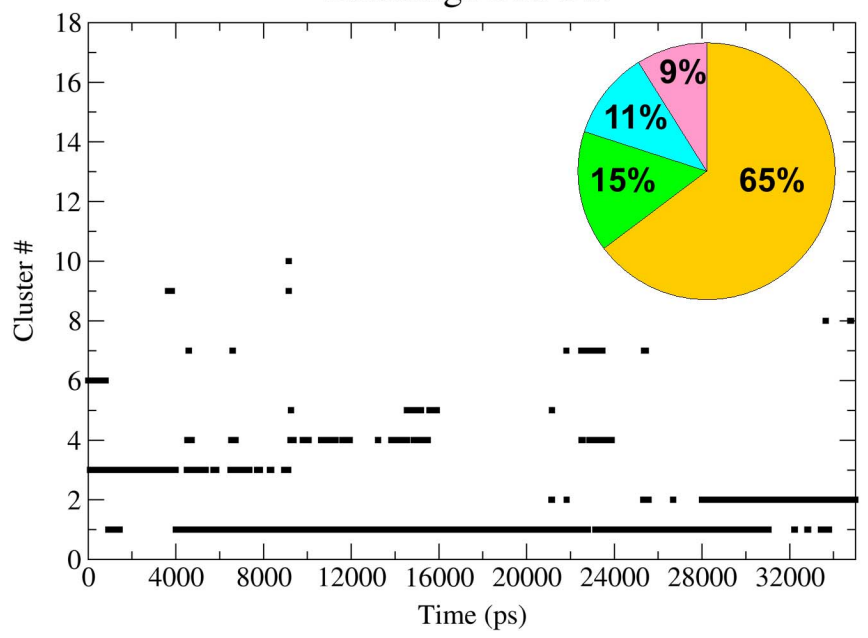

Exchange 560-581

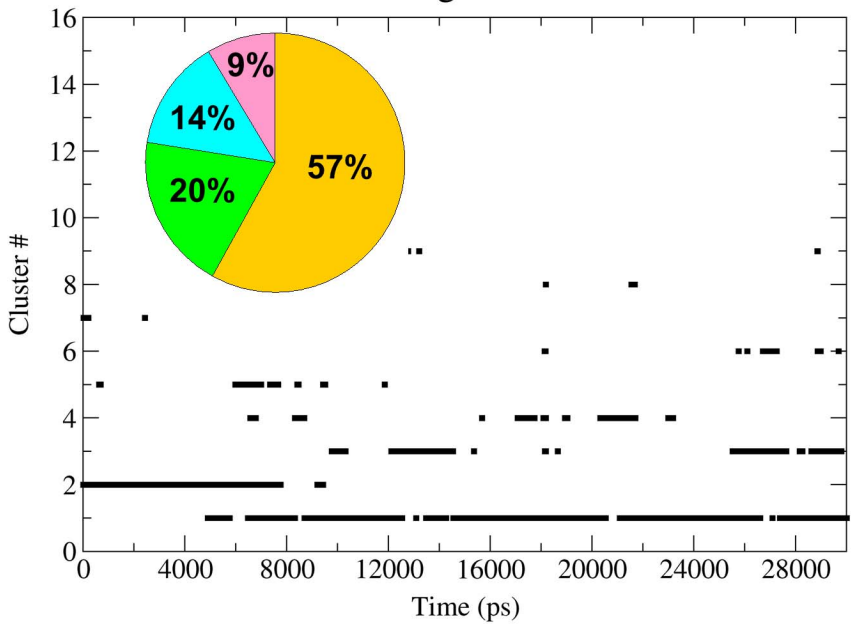

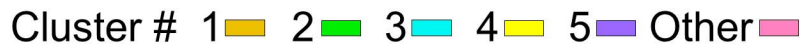

Figure 5. Cluster analysis of WT, C583S and two exchange simulations. The figure depicts the results by two presentations for the four simulations as indicated in each panel. The main panel in each frame shows the transitions between clusters (identified on the ordinate by numbers) along the trajectories. The relative fraction of the dominant clusters is presented by the colored histogram. The clusters are numbered sequentially from the most $(\# 1)$ to the less popular. The identity of the system under analysis is marked in the frames.

doi:10.1371/journal.pone.0059175.g005 
protein in a specific structure without preventing it from sampling other conformations.

\section{The C581S Mutation and the Derived Disulfide Exchange Reaction}

As reported previously, any mutation in one of the cysteine residues in the native pair $\mathrm{Cys}^{567}{ }^{5} \mathrm{Cys}^{581}$ significantly reduces the activation of integrin; thus, it was suggested that this disulfide bond makes a structural contribution to the ability of the protein to function normally in the activation mechanism. Moreover, the C581S mutation resulted not only in reduced expression of the integrin, but, more importantly, in a totally inactive status [29]. For that reason it was interesting to mutate C581 to serine in-silico, leaving its Cys567 partner with a free $\mathrm{SH}$ group (Table 1, number 6 ), and to examine what structural conformation the protein undergoes, besides the fact that a disulfide exchange with Cys560 is no longer possible.

According to the crystal state, residue Cys567 is only $\sim 4.5 \AA$ from the Cys ${ }^{575}$ - Cys ${ }^{586}$ disulfide pair (Figure 1), suggesting that a disulfide exchange is feasible. During the C581S simulations the distances between sulfur atoms of Cys567 from Cys575 or Cys586 were maintained with an average value of $3.7 \AA$ (see Figure S4). None of the other cysteine residues came to a covalent distance with Cys567. This suggests that the free Cys567 residue can also participate in, or initiate, a set of disulfide rearrangements, where the $\mathrm{Cys}^{575} \mathrm{Cys}^{586}$ bond is the preferred target. To check the structural effect of this event we connected the free thiol of Cys567 to the sulfur atom of Cys586 and initiated the simulation from the last snapshots of C581S simulation. The outcome of cluster analysis of C581S mutant and Cys ${ }^{567}$-Cys ${ }^{586}$ exchange, simulated for an additional $40 \mathrm{~ns}$, is presented in Figure 6 and in Fig. S5.

For the C581S mutant, the trajectory is characterized by a dominance of the first and the second cluster, which together consist of $\sim 2 / 3$ of the total states. Once the free cysteine (Cys567) was linked with Cys586 (the nearest member of the Cys575Cys586 pair) a very stable structure emerged, where the first cluster was almost $1.6 k_{\mathrm{B}} \mathrm{T}$ more stable than the second (Fig. 6). In comparison with the other simulations described in this study, this mutant exhibits a clear preferential convergence of structure. The trajectory revealed a distinctly dominant structure, generated during the early phase of the simulations, persisting $(80 \%$ of the total number of states) until the simulation was terminated. These data indicate that in the C581S mutant the thiol/disulfide exchange event locks the protein in one defined conformational space.

\section{Discussion}

Disulfide isomerization in vivo produces structural isomers with the same oxidation state; thus, traditional biochemistry techniques have serious limitations in exposing specific redox sites. These approaches usually freeze thiol/disulfide exchange reactions at specific times and analyze the products by high-performance liquid chromatography, electrophoresis or NMR spectroscopy $[45,46]$. Recently, a single-molecule force spectroscopy was used to monitor disulfide bond reduction and isomerization $[47,48]$. However, the experimental results are frequently difficult to interpret and specific cysteine residues, prone to such an exchange, remain to be defined unambiguously. Therefore, direct identification of thiol/disulfide exchange events in complex proteins and, specifically, in the $\alpha \operatorname{IIb} \beta 3$ is currently possible only with computational methods, the first attempt of which is presented here.

The lower leg of the $\beta 3$ subunit is built of a sequence of rigid elements: the four I-EGF domains and the $\beta$-tail are all $\beta$-sheet structures that are stabilized by internal disulfide bonds. Despite the apparent stability of the structural elements, the $\alpha \operatorname{IIb} \beta 3$ receptor experiences a massive structural fluctuation during its activation. The presence of multiple disulfides render the protein to be very rigid and resistant to deformation [49]. Thus, the introduction of free thiols that can initiate a sequence of SH/S-S replacements and lead to structural modulation seems to be necessary. In the present study we evaluated the possibility of disulfide bond exchange and to what extent the rearrangement of the disulfide network can contribute to the flexibility and the
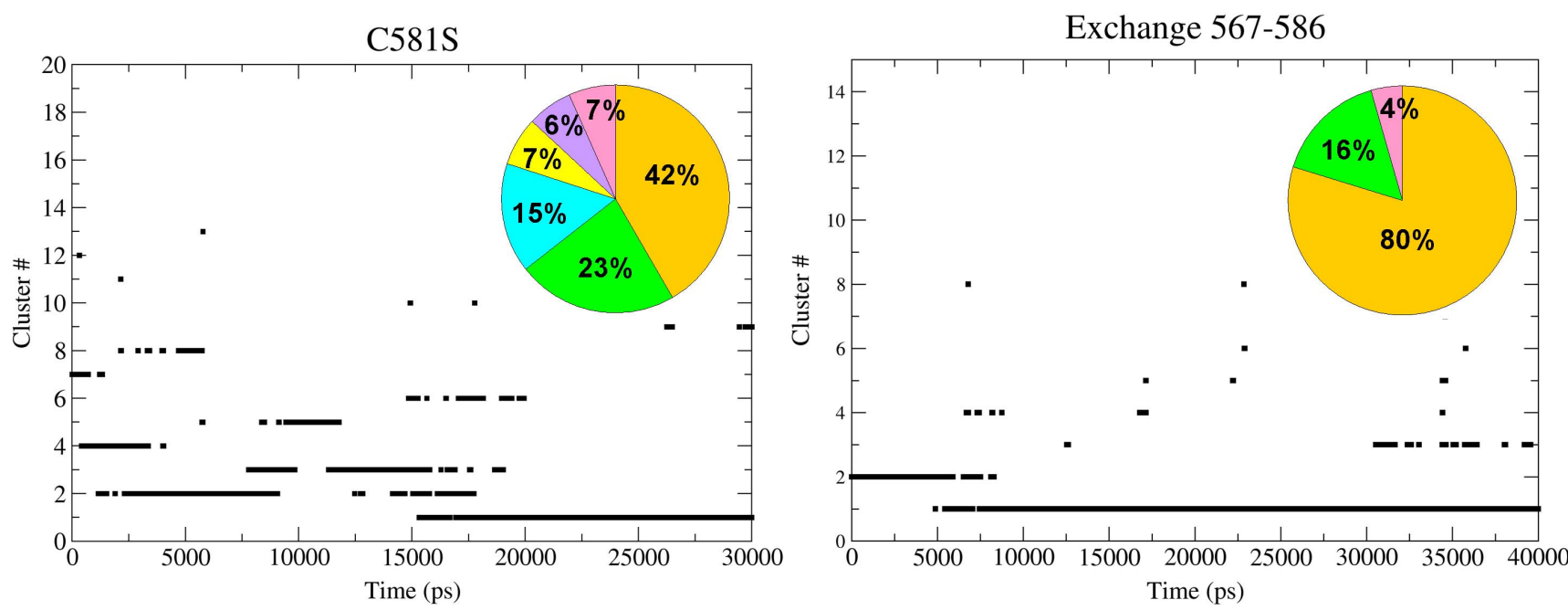

Cluster \# 1ロ $2 \square 3 \square 4 \square 5 \square$ Other $\square$

Figure 6. Cluster analysis of C581S and Cys ${ }^{567}-C_{y s}{ }^{586}$ simulations. Cluster analysis of C581S (left) and Cys ${ }^{567}$-Cys ${ }^{586}$ (right) disulfide simulations. The figure depicts the results by two presentations for the two representative simulations as indicated in each panel. The main frame shows the transitions between clusters (identified on the ordinate by numbers) along the trajectories. The relative fraction of the dominant clusters is presented by the colored histogram. The clusters are numbered sequentially from the most (\#1) to the less popular. The identity of the system under analysis is marked in the frames.

doi:10.1371/journal.pone.0059175.g006 
stability of the protein. For this purpose we introduced in-silico mutations representing well experimentally documented mutants, in which a single cysteine of a disulfide bond was replaced either by serine (C583S and C581S) or by arginine (C560R) and monitored the proximity of the partner-free thiol to the other cysteine pairs by MD. Whenever sulfur atoms came within a range where a covalent bond may be formed, we performed an in-silico thiol/disulfide exchange and simulated these new structures, focusing on the structural effects of these rearrangements.

The starting point of the study was the Cys ${ }^{560}-\mathrm{Cys}{ }^{583}$ bond, which experimentally leads to two, antipodal scenarios; either a constitutively active protein (C560R) or an almost normal activation status with reduced expression (C583S) [22,23,29]. This indicates that the single disruption of a disulfide bond could lead to two different structural deformations. We allowed both mutants to exercise an unbiased MD run, in the presence of explicit solvent and physiologic ionic strength, focusing on the structural impact of these mutations. Comparison of the trajectory with that of the WT protein indicated that during the simulation, the C583S mutant experienced a massive conformational change. However, none of the domains were distorted, indicating that the main conformational changes are an outcome of inter-domain motions, whereas the sub-elements retained their rigidity. It is of interest to point out that the mechanical stability of multi-domain proteins exhibits a lower resistance to relative motion of the domains compared to deformation of the domains themselves [49].

A mutation of a cysteine residue, which participates in a disulfide bond, not only breaks the link between two cysteine residues but also releases the second cysteine, so it can participate in disulfide exchange reaction with other disulfide bonds that it encounters during structural fluctuation. In the C583S simulation, the sulfhydryl moiety of Cys560, released from the disulfide bond, was free to move around its immediate vicinity. Based on the crystal structure, Cys560 has two possible partners for disulfide exchange; $\mathrm{Cys}^{567}-\mathrm{Cys}^{581}$ or Cys ${ }^{549}-\mathrm{Cys}{ }^{558}$ which are located at almost equal proximity (Fig. 1). However, the unbound Cys560 did not scan the whole space nearby but tended to favor a certain disulfide, the $\mathrm{Cys}^{567}$-Cys ${ }^{581}$ bond (Fig. 4, left panel, Fig. S3). In about $44 \%$ of the total number of frames, from 7 different simulations, the free thiol group approached the sulfur atoms, to such proximity that can allow attraction forces to eventually produce an exchange reaction. This region-specificity is probably imposed by the rigidity of the domain gained by the high content of $\beta$-strands in this protein region, which limits the ability of the structure to sample a large conformation landscape. In contrast with the scenario observed for the C583S simulation, the simulation of the $\mathrm{C} 560 \mathrm{R}$ mutant failed to converge into a stable structure where the Cys583 moiety did not initiate any attack on any other disulfide pair. It should be emphasized that the constitutive activation of the protein, caused by the mutation C560R, is not specific to arginine substitution, it is common to 11 other amino acid replacements of Cys560 [50]. Thus, simulation of C560R should exhibit a similar result as with the other mutations, which can also imply for the insignificance of the sidechain property in this critical condition. As mentioned above, it should also be stressed that in-vitro experiments done by MorCohen et al. [23,29] demonstrated that, in contradiction to the constitutively active Cys560 substitutions, the C583S mutation has nearly normal activation status. By combining these in vitro results with the MD simulations performed in this study, it can be suggested that the mutated C583S integrin is spared from gaining the permanent over-activated status, due to conformational changes initiated by the attack of the unbound free Cys560 to the $\mathrm{Cys}^{567}{ }^{5 y \mathrm{C}^{581}}$ pair, thus enabling a thiol/disulfide exchange which alters the rearrangement of disulfide bonds.

In accordance with the established role of disulfide bond reduction during integrin activation, we reduced the Cys ${ }^{567}$ $\mathrm{Cys}^{581}$ disulfide bond and connected the free Cys560 to either Cys567 or Cys581. Both structures, containing either a Cys ${ }^{560}$ $\mathrm{Cys}^{567}$ or $\mathrm{Cys}^{560}-\mathrm{C} y s^{581}$ bond, were subjected to further MD simulations. Once the new disulfide bonds were formed, the protein acquired new conformations that were evaluated by their relative stability using cluster analysis of the trajectories. In all cases we could identify a dominant structure which was in very rapid equilibrium with other states. This flexibility could lead to other disulfide rearrangements in nature and be the key to the integrin activation process. The rearrangements between $\mathrm{Cys}^{560}$ $\mathrm{Cys}^{583}$ and $\mathrm{Cys}^{567}{ }^{5 y s}{ }^{581}$ bonds, tested by MD in this study, are also in agreement with an alternative disulfide pairing of $\alpha \operatorname{IIb} \beta 3$ suggested by protein digestion assay, which connects Cys560 to Cys567 [27], though this proposed Cys ${ }^{560}-\mathrm{Cys}^{567}$ bond was not further confirmed by protein crystallization studies. Thus, we suggest that the Cys ${ }^{560}-\mathrm{Cys}^{567}$ is not a permanent bond, but may be one phase in the multistage activation process of the solvated $\alpha \operatorname{IIb} \beta 3$ protein.

In the crystal structure the Cys ${ }^{567}-\mathrm{Cys}^{581}$ and the Cys ${ }^{575}$ $\mathrm{Cys}^{586}$ bonds are located in almost covalent distance from each other. To check whether this proximity would persist in a dynamic system, we simulated C581S mutant and monitored the distance between the created free Cys567 to other disulfide bonds. Not surprisingly, the proximity to the $\mathrm{Cys}^{575}-\mathrm{Cys}^{586}$ bond was maintained and even increased. To follow this tendency we performed a thiol/disulfide exchange reaction by linking free Cys567 to Cys586. The resulting product strongly prefers one defined conformational state, spending $80 \%$ of simulation time in a specific structure, thereby losing the sampling ability of other conformations. Due to the native proximity of the free Cys567 to the Cys ${ }^{575}-\mathrm{Cys}^{586}$ bond and to the structural consequence of the exchange with Cys586, we propose that the C581S mutation forces the above exchange, thus bringing the protein to a rigid structure, locked in one specified conformation that is not able to proceed to the activation process. This suggestion agrees with the observation that each distortion of the native $\mathrm{Cys}^{567}-\mathrm{Cys}^{581}$ bond significantly reduces the activation of $\alpha \operatorname{IIb} \beta 3$ [23,29].

Based on our simulations, we propose that the ability to produce a disulfide exchange reaction between Cys560 (from the Cys ${ }^{560}$ $\mathrm{Cys}^{583}$ pair) with Cys567 from the next pair of cysteine residues $\left(\mathrm{Cys}^{567}-\mathrm{Cys}^{581}\right)$ is one essential step towards normal activity of the protein. Thus, any mutation in Cys560 or in the Cys ${ }^{567}-\mathrm{Cys}^{581}$ pair that interrupts this important disulfide exchange reaction would cause a severe failure in the activation mechanism of the integrin, as was found experimentally.

Many studies [22,23,29,50-52] have shown that specific mutations in the cysteine residues of $\beta 3$ give rise to a constitutively active receptor. Basing our reasoning on lack of thiol/disulfide exchange ability, as exemplified by the C560R, we could hypothesize that at least some of these mutations produce a rigid structure, not capable of rearrangement, which is, therefore, fixed in a constitutively active state.

It is clear that thiol/disulfide exchange plays an important role in $\alpha \operatorname{IIb} \beta 3$ and the closely related $\alpha_{\mathrm{V}} \beta 3$ activation and, indeed, unpaired cysteine thiols appear in the $\beta 3$ subunit of these integrins upon activation [53,54]. Considering the large number of disulfide bonds, the number of resulting conformations can increase exponentially [47]. However, as presented in this study, the outcome of disulfide rupture is similar to a collapse of domino blocks, following a given pattern. Apparently, the initiation of a 
single sulfhydryl moiety can generate a unique sequence of sulfhydryl exchange events leading to new stable conformations, one after the other, and resulting in a gradual distortion of the lower leg section of the $\beta 3$ subunit of the integrin. The cysteine residues investigated in this study are so close on the primary sequence of the protein that the $\beta$-sheet structures need to pucker to accommodate the disulfides. This puckering is believed to cause a significant strain on the bonds $[55,56]$. There is evidence that locally tolerated stress is important for protein function and that high torsional energies have an enhanced likelihood of reduction [57]. Therefore, the described cysteine residues may have a functional redox role. Recent research has shown that surface thiols in $\alpha$ IIb $\beta 3$ are exposed during platelet activation and, more importantly, different populations of the integrin exist on platelets based on differential labeling of thiols $[31,58]$. These allosteric thiols may participate in the thiol/disulfide exchange reactions, leading to the integrin activation or deactivation processes. Although crystallography has not identified free thiols in $\alpha \operatorname{IIb} \beta 3$, it is possible that their oxidation to disulfide bonds occurs in the expression, purification or crystallization procedures.

\section{Conclusions}

The results presented in this study demonstrate the possibility of disulfide exchange in the dynamic system of cysteine mutants of $\alpha \operatorname{IIb} \beta 3$ integrin and show the structural consequences of these replacements. We show that the conformational space that the protein may scan, once a disulfide bond is cleaved, is rather limited and the structural fluctuations tend to converge into a "predestined" conformation. We suggest that overly rigid structures may not be able to go through a thiol/disulfide exchange and, therefore, give rise to an unnatural state of activation, e.g. a constitutively active or inactive protein. We propose that specific redox sites reported in the present study may play a crucial role in the overall conformational changes of $\alpha \operatorname{IIb} \beta 3$ integrin during its activation and bidirectional signaling.

\section{Supporting Information}

Figure S1 Superposition of the WT protein and the C560R mutant. Superposition of the most favorable conformation of a simulated WT protein (red) vs. the most favorable conformation of the simulated C560R mutant (light blue). The

\section{References}

1. Coller BS, Shattil SJ (2008) The GPIIb/IIIa (integrin $\alpha$ IIb $\beta 3$ ) odyssey: a technology-driven saga of a receptor with twists, turns, and even a bend. Blood 112: 3011-3025.

2. Oxvig C, Lu C, Springer TA (1999) Conformational changes in tertiary structure near the ligand binding site of an integrin I domain. Proc Natl Acad Sci U S A 96: 2215-2220.

3. Shattil SJ, Kashiwagi H, Pampori N (1998) Integrin signaling: the platelet paradigm. Blood 91: 2645-2657.

4. Eng ET, Smagghe BJ, Walz T, Springer TA (2011) Intact alphaIIbbeta3 integrin is extended after activation as measured by solution X-ray scattering and electron microscopy. J Biol Chem 286: 35218-35226.

5. Takagi J, Petre BM, Walz T, Springer TA (2002) Global conformational rearrangements in integrin extracellular domains in outside-in and inside-out signaling. Cell 110: 599-611.

6. Xiao T, Takagi J, Coller BS, Wang JH, Springer TA (2004) Structural basis for allostery in integrins and binding to fibrinogen-mimetic therapeutics. Nature 432: 59-67.

7. Zhu J, Boylan B, Luo BH, Newman PJ, Springer TA (2007) Tests of the extension and deadbolt models of integrin activation. J Biol Chem 282: 11914 11920.

8. Adair BD, Xiong JP, Maddock C, Goodman SL, Arnaout MA, et al. (2005) Three-dimensional EM structure of the ectodomain of integrin $\{$ alpha $\}$ V $\{$ beta 3 in a complex with fibronectin. J Cell Biol 168: 1109-1118.

9. Xiong JP, Stehle T, Goodman SL, Arnaout MA (2003) New insights into the structural basis of integrin activation. Blood 102: 1155-1159. upper panels introduce the differences in each domain (I-EGF1 to $\beta$-tail as indicated); the lower panel shows the entire penta-domain structure.

(TIF)

Figure S2 RMSD of the backbone atoms from their initial coordinates as a function of time. The average and S.D. of the last $20 \mathrm{~ns}$ (in red) are presented above every frame. (A) WT, (B) C560R and (C) C583S.

(TIF)

Figure S3 The minimum distance between the free thiol Gys560 (in the G583S simulation) and the disulfide pair $\mathbf{C y s}^{\mathbf{5 4 9}}-\mathbf{C y s}^{\mathbf{5 5 8}}$ in I-EGF3. The distance was calculated between the sulfur atoms.

(TIF)

Figure S4 The minimum distance between the free thiol Cys567 and Cys586 in the three C581S simulations. The distance was calculated between the sulfur atoms.

(TIF)

Figure S5 Gluster analysis of simulations number $2 \& 3$ of the G581S mutation (left \& right respectively). The figure depicts the results by two presentations for the two simulations. The main frame shows the transitions between clusters (identified on the ordinate by numbers) along the trajectories. The relative fraction of the dominant clusters is presented by the colored histogram. The clusters are numbered sequentially from the most $(\# 1)$ to the less popular.

(TIF)

Table S1 List of C583S simulations. (DOCX)

\section{Acknowledgments}

We thank Dr. Ronit Mor-Cohen for critical reading of this manuscript and Dr. Nurit Rosenberg for helpful discussions.

\section{Author Contributions}

Conceived and designed the experiments: YE LL EZ EN MG YT. Performed the experiments: LL EZ. Analyzed the data: LL EZ. Wrote the paper: LL EN MG YT YE.
10. Ye F, Liu J, Winkler H, Taylor KA (2008) Integrin alpha IIb beta 3 in a membrane environment remains the same height after Mn2+ activation when observed by cryoelectron tomography. J Mol Biol 378: 976-986.

11. Essex DW (2004) The role of thiols and disulfides in platelet function. Antioxid Redox Signal 6: 736-746.

12. Jiang XM, Fitzgerald M, Grant CM, Hogg PJ (1999) Redox control of exofacial protein thiols/disulfides by protein disulfide isomerase. J Biol Chem 274: 24162423.

13. Jordan PA, Gibbins JM (2006) Extracellular disulfide exchange and the regulation of cellular function. Antioxid Redox Signal 8: 312-324.

14. Lahav J, Jurk K, Hess O, Barnes MJ, Farndale RW, et al. (2002) Sustained integrin ligation involves extracellular free sulfhydryls and enzymatically catalyzed disulfide exchange. Blood 100: 2472-2478.

15. Yan B, Smith JW (2001) Mechanism of integrin activation by disulfide bond reduction. Biochemistry 40: 8861-8867.

16. O'Neill S, Robinson A, Deering A, Ryan M, Fitzgerald DJ, et al. (2000) The platelet integrin alpha IIbbeta 3 has an endogenous thiol isomerase activity. J Biol Chem 275: 36984-33690.

17. Yan B, Smith JW (2000) A redox site involved in integrin activation. J Biol Chem 275: 39964-39972.

18. Beglova N, Blacklow SC, Takagi J, Springer TA (2002) Cysteine-rich module structure reveals a fulcrum for integrin rearrangement upon activation. Nat Struct Biol 9: 282-287.

19. Chen P, Melchior C, Brons NH, Schlegel N, Caen J, et al. (2001) Probing conformational changes in the I-like domain and the cysteine-rich repeat of human beta 3 integrins following disulfide bond disruption by cysteine 
mutations: identification of cysteine 598 involved in alphaIIbbeta3 activation. J Biol Chem 276: 38628-38635.

20. D'Andrea G, Colaizzo D, Vecchione G, Grandone E, Di Minno G, et al. (2002) Glanzmann's thrombasthenia: identification of 19 new mutations in 30 patients. Thromb Haemost 87: 1034-1042.

21. Milet-Marsal S, Breillat C, Peyruchaud O, Nurden P, Combrie R, et al. (2002) Two different beta3 cysteine substitutions alter alphaIIb beta3 maturation and result in Glanzmann thrombasthenia. Thromb Haemost 88: 104-110.

22. Ruiz C, Liu CY, Sun QH, Sigaud-Fiks M, Fressinaud E, et al. (2001) A point mutation in the cysteine-rich domain of glycoprotein (GP) IIIa results in the expression of a GPIIb-IIIa (alphaIIbbeta3) integrin receptor locked in a highaffinity state and a Glanzmann thrombasthenia-like phenotype. Blood 98: 24322441.

23. Mor-Cohen R, Rosenberg N, Einav Y, Zelzion E, Landau M, et al. (2012) Unique disulfide bonds in epidermal growth factor (EGF) domains of beta3 affect structure and function of alphaIIbbeta 3 and alphavbeta3 integrins in different manner. J Biol Chem 287: 8879-8891.

24. Xiong JP, Mahalingham B, Alonso JL, Borrelli LA, Rui X, et al. (2009) Crystal structure of the complete integrin alphaVbeta3 ectodomain plus an alpha/beta transmembrane fragment. J Cell Biol 186: 589-600.

25. Zhu J, Luo BH, Xiao T, Zhang C, Nishida N, et al. (2008) Structure of a complete integrin ectodomain in a physiologic resting state and activation and deactivation by applied forces. Mol Cell 32: 849-861.

26. Takagi J, Beglova N, Yalamanchili P, Blacklow SC, Springer TA (2001) Definition of EGF-like, closely interacting modules that bear activation epitopes in integrin beta subunits. Proc Natl Acad Sci U S A 98: 11175-11180.

27. Calvete JJ, Henschen A, Gonzalez-Rodriguez J (1991) Assignment of disulphide bonds in human platelet GPIIIa. A disulphide pattern for the beta-subunits of the integrin family. Biochem J 274 (Pt 1): 63-71.

28. Xiong JP, Stehle T, Diefenbach B, Zhang R, Dunker R, et al. (2001) Crystal structure of the extracellular segment of integrin alpha Vbeta3. Science 294: 339-345.

29. Mor-Cohen R, Rosenberg N, Landau M, Lahav J, Seligsohn U (2008) Specific cysteines in beta3 are involved in disulfide bond exchange-dependent and independent activation of alphaIIbbeta3. J Biol Chem 283: 19235-11944.

30. Gaillard T, Dejaegere A, Stote RH (2009) Dynamics of beta3 integrin I-like and hybrid domains: insight from simulations on the mechanism of transition between open and closed forms. Proteins 76: 977-994.

31. Mansour W, Einav Y, Hauschner H, Koren A, Seligsohn U, et al. (2011) An alphaIIb mutation in patients with Glanzmann thrombasthenia located in the Nterminus of blade 1 of the beta-propeller (Asn2Asp) disrupts a calcium binding site in blade 6. J Thromb Haemost 9: 192-200.

32. Provasi D, Murcia M, Coller BS, Filizola M (2009) Targeted molecular dynamics reveals overall common conformational changes upon hybrid domain swing-out in beta3 integrins. Proteins 77: 477-489.

33. Puklin-Faucher E, Gao M, Schulten K, Vogel V (2006) How the headpiece hinge angle is opened: New insights into the dynamics of integrin activation. J Cell Biol 175: 349-360.

34. Puklin-Faucher E, Vogel V (2009) Integrin activation dynamics between the RGD-binding site and the headpiece hinge. J Biol Chem 284: 36557-36568.

35. Guex N, Peitsch MC (1997) SWISS-MODEL and the Swiss-PdbViewer: an environment for comparative protein modeling. Electrophoresis 18: 2714-2723.

36. Lindahl E, Hess B, Van Der Spoel D (2001) GROMACS 3.0: a package for molecular simulation and trajectory analysis. Journal of Molecular Modelling: 306-317.

37. Van Der Spoel D, Lindahl E, Hess B, Groenhof G, Mark AE, et al. (2005) GROMACS: fast, flexible, and free. J Comput Chem 26: 1701-1718.
38. Oostenbrink C, Villa A, Mark AE, van Gunsteren WF (2004) A biomolecular force field based on the free enthalpy of hydration and solvation: the GROMOS force-field parameter sets 53A5 and 53A6. J Comput Chem 25: 1656-1676.

39. Berendsen HJC, Postma JPM, Gunsteren WFV, Hermans J (1981) Interaction models for water in relation to protein hydration. Intermolecular Forces 11: 331-338.

40. Berendsen HJC, Postma JPM, Van Gunsteren WF, DiNola A, Haak JR (1984) Molecular dynamics with coupling to an external bath. The Journal of Chemical Physics 81: 3684-3691.

41. Hess B, Bekker H, Berendsen HJC, Fraaije JGEM (1997) LINCS: A linear constraint solver for molecular simulations. Journal of Computational Chemistry 18: $1463-1472$.

42. Essmann U, Perera L, Berkowitz ML, Darden TA, Lee H, et al. (1995) A Smooth Particle Mesh Ewald method. Journal of Medical Physics 103: 85778592.

43. Humphrey W, Dalke A, Schulten K (1996) VMD: visual molecular dynamics. J Mol Graph 14: 33-38, 27-28.

44. Daura X, van Gunsteren WF, Mark AE (1999) Folding-unfolding thermodynamics of a beta-heptapeptide from equilibrium simulations. Proteins 34: 269280.

45. Gilbert HF (1995) Thiol/disulfide exchange equilibria and disulfide bond stability. Methods Enzymol 251: 8-28.

46. Wedemeyer WJ, Welker E, Narayan M, Scheraga HA (2000) Disulfide bonds and protein folding. Biochemistry 39: 4207-4216.

47. Alegre-Cebollada J, Kosuri P, Rivas-Pardo JA, Fernandez JM (2011) Direct observation of disulfide isomerization in a single protein. Nat Chem 3: 882-887.

48. Wiita AP, Ainavarapu SR, Huang HH, Fernandez JM (2006) Force-dependent chemical kinetics of disulfide bond reduction observed with single-molecule techniques. Proc Natl Acad Sci U S A 103: 7222-7227.

49. Sikora M, Cieplak M (2011) Mechanical stability of multidomain proteins and novel mechanical clamps. Proteins 79: 1786-1799.

50. Kamata T, Ambo H, Puzon-McLaughlin W, Tieu KK, Handa M, et al. (2004) Critical cysteine residues for regulation of integrin alphaIlbbeta 3 are clustered in the epidermal growth factor domains of the beta3 subunit. Biochem J 378: 1079-1082.

51. Butta N, Arias-Salgado EG, Gonzalez-Manchon C, Ferrer M, Larrucea S, et al. (2003) Disruption of the beta3 663-687 disulfide bridge confers constitutive activity to beta3 integrins. Blood 102: 2491-2497.

52. Mor-Cohen R, Rosenberg N, Peretz H, Landau M, Coller BS, et al. (2007) Disulfide bond disruption by a beta 3-Cys549Arg mutation in six Jordanian families with Glanzmann thrombasthenia causes diminished production of constitutively active alpha IIb beta 3. Thromb Haemost 98: 1257-1265.

53. Essex DW, Li M (2003) Redox control of platelet aggregation. Biochemistry 42: 129-136.

54. Swiatkowska M, Szymanski J, Padula G, Cierniewski CS (2008) Interaction and functional association of protein disulfide isomerase with alphaVbeta3 integrin on endothelial cells. Febs J 275: 1813-1823.

55. Schmidt B, Ho L, Hogg PJ (2006) Allosteric disulfide bonds. Biochemistry 45: 7429-7433.

56. Wouters MA, Lau KK, Hogg PJ (2004) Cross-strand disulphides in cell entry proteins: poised to act. Bioessays 26: 73-79.

57. Wouters MA, Fan SW, Haworth NL (2010) Disulfides as redox switches: from molecular mechanisms to functional significance. Antioxid Redox Signal 12: 5391.

58. Manickam N, Ahmad SS, Essex DW (2011) Vicinal thiols are required for activation of the $\alpha \operatorname{IIb} \beta 3$ platelet integrin. Journal of Thrombosis and Haemostasis 9: 1207-1215. 